\title{
Successful treatment of metastatic urothelial carcinoma arising in a transplanted renal allograft with paclitaxel, cisplatin, and gemcitabine combination therapy: a case report
}

Yasuyuki Kojima ${ }^{1 *}$, Yuko Takahi ${ }^{1}$, Naotsugu Ichimaru ${ }^{2}$, Masayoshi Okumi ${ }^{3}$, Shiro Takahara ${ }^{2}$ and Norio Nonomura ${ }^{3}$

\begin{abstract}
Background: For locally advanced or metastatic urothelial carcinoma, cisplatin-based chemotherapy is the standard regimen. Nevertheless, almost all responding patients experience recurrence within the first year. When patients who have received prior cisplatin-based therapy become resistant, combination therapy with gemcitabine and paclitaxel has been reported. Few published case reports have addressed the utility of paclitaxel/cisplatin/gemcitabine combination therapy as second-line chemotherapy for advanced or metastatic urothelial carcinoma. This is the first report describing paclitaxel/cisplatin/gemcitabine combination therapy for metastatic urothelial carcinoma arising in a transplanted renal allograft and leading to a successful outcome.
\end{abstract}

Case presentation: We present a case of metastatic urothelial carcinoma of a renal allograft in a 32-year-old Japanese man with a history of kidney transplantation ten years prior. Because the patient's serum creatinine increased, hemodialysis was resumed, and the surgical allograft was removed. Multiple lung metastases were resistant to gemcitabine/cisplatin adjuvant chemotherapy, so paclitaxel/cisplatin/gemcitabine combination chemotherapy was instituted. After paclitaxel/cisplatin/gemcitabine chemotherapy, all pulmonary metastatic tumors disappeared. The patient has survived without disease progression for more than four years since treatment.

Conclusion: Paclitaxel/cisplatin/gemcitabine combination therapy may be effective and lead to a survival advantage in patients with locally advanced or metastatic urothelial carcinoma when used as second-line chemotherapy following cisplatin-based therapy. However, further investigations may be required to confirm and evaluate the significance of this treatment.

Keywords: Urothelial carcinoma, Paclitaxel, Cisplatin, Gemcitabine, Kidney transplantation, Hemodialysis

\section{Background}

For locally advanced or metastatic urothelial carcinoma (UC), methotrexate/vinblastine/doxorubicin/cisplatin (MVAC) [1] or gemcitabine/cisplatin (GC) [2] combination chemotherapy represents the standard regimen. However when patients who have received prior cisplatinbased therapy become resistant, there is no standard regimen for second-line chemotherapy.

\footnotetext{
* Correspondence: yakojima@soryu.jp

${ }^{1}$ Department of Urology, Inoue Hospital, 16-17 Enoki-Cho, Suita 564-0053,

Osaka, Japan

Full list of author information is available at the end of the article
}

In general, malignant tumors occur more frequently in renal transplant recipients receiving immunosuppressive therapy. These malignancies are often more aggressive and are associated with a poor prognosis [3-5].

We report a case of metastatic UC of a renal allograft in a patient who showed long-term recurrence-free survival after being treated with a combination of paclitaxel, cisplatin, and gemcitabine (PCG).

\section{Case presentation}

A 32-year-old Japanese man presented with gross hematuria. He had undergone a kidney transplant ten years earlier for end-stage kidney disease with focal

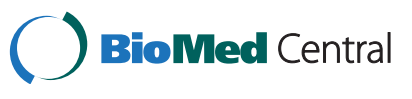


segmental glomerulosclerosis at that time, he received a living kidney allograft from his 51-year-old father. On presentation, an abdominal computed tomography (CT) scan revealed a mass of approximately $4 \mathrm{~cm}$ in the allograft collecting system. Chest CT showed multiple lung metastases. The results of annual screening with abdominal CT and ultrasonography had previously been normal. The patient's Eastern Cooperative Oncology Group (ECOG) performance status was 1 at this time.

Ureteroscopic biopsy was performed, and histopathology showed a grade 3, urothelial carcinoma. The clinical diagnosis was renal pelvis cancer of stage T2N0M1 according to the TNM classification of Malignant Tumors (7th edition).

After hemodialysis was resumed, we planned GC neoadjuvant chemotherapy. Gemcitabine (GEM) was administered at $1,000 \mathrm{mg} / \mathrm{m}^{2}$ on non-dialysis days 1,8 , and 15. Cisplatin (CDDP) was given intravenously at $35 \mathrm{mg} /$ $\mathrm{m}^{2}$ on dialysis day 2 . This regimen was repeated every four weeks.

However, the tumor showed progression following two courses of GC treatment (Figure 1), so we decided to perform renal allograftectomy. Histopathological examination confirmed a grade 3 invasive UC with final staging of pT3 (Figure 2).

After the surgery, one course of postoperative GC treatment was administered. Evaluation with chest $\mathrm{CT}$ according to RECIST guidelines (version 1.1) showed increased size of the pulmonary metastatic tumors, judged as progressive disease (PD). As a result, paclitaxel/ cisplatin/gemcitabine (PCG) combination therapy was started. The PCG protocol consisted of GEM 1,000 mg/m ${ }^{2}$ and paclitaxel (PTX) $80 \mathrm{mg} / \mathrm{m}^{2}$ on non-dialysis days 1 and 8 plus CDDP $35 \mathrm{mg} / \mathrm{m}^{2}$ on dialysis day 2 . This course was repeated every three weeks.

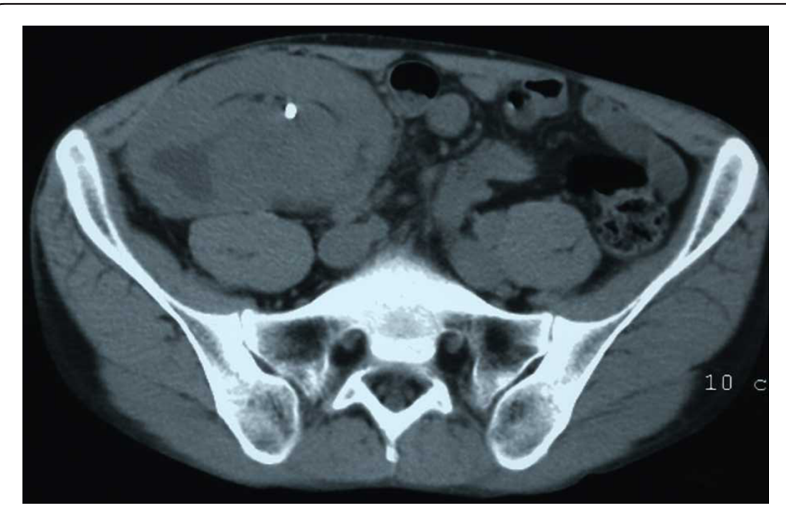

Figure 1 Abdominal computed tomography (CT) scan of the patient. Abdominal CT scan prior to surgery showed a mass measuring $6.4 \times 3.7 \mathrm{~cm}$ in the transplanted kidney. A double-J ureteral stent was placed.
After two courses of PCG, pulmonary metastatic tumors showed a $45 \%$ size reduction on chest $\mathrm{CT}$, judged as a partial response (PR). After seven courses of PCG chemotherapy, CT demonstrated the disappearance of all pulmonary metastatic tumors, judged as a complete response (CR) (Figure 3). Toxicity was evaluated using the Common Terminology Criteria for Adverse Events (CTCAE) as a grade 2 neutropenia. There was no severe toxicity. Currently, the patient has been tumor-free for 54 months following treatment.

\section{Discussion}

The overall incidence of de novo malignancies after renal transplantation is much higher than that in the general population because the former patients receive immunosuppressive therapy [6]. The risk of post-transplant UC is higher among patients with aristolochic acid nephropathy (AAN) (also called Chinese-herb nephropathy) [7] and those among patients with analgesic nephropathy (AN) because of prior analgesic abuse [8]. Several cases of UC (bladder, ureter, and renal pelvis involvement) have been reported in renal transplant patients without AAN or AN $[9,10]$. Renal pelvis cancer following a kidney transplantation almost occurs in the native kidney. The cause is unknown, but post-transplant UC of a renal allograft rarely occurs in the long-term postoperative period [11-13]. To detect malignancies, an intensive follow-up regimen including urine cytology should be adopted after renal transplantation [14].

MVAC combination chemotherapy is the standard regimen for locally advanced or metastatic UC [1]. A combination of gemcitabine and cisplatin (GC) has shown similar survival times compared with MVAC but with better safety [2]. GC is now considered to be another standard regimen for patients with locally advanced or metastatic UC.

However, an effective treatment strategy has not yet been established for patients who show resistance to initial treatment such as GC therapy. Some authors have reported the efficacy of gemcitabine/paclitaxel (GEM/ PTX) combination therapy and the PCG combination therapy that we evaluated in our study [15-17].

The overall response rate (complete or partial) with GEM/ PTX treatment following initial cisplatin or carboplatinbased therapy ranges from 60 to $66.7 \%[15,16]$. With PCG therapy, an overall response rate of $77.6 \%$ and median survival time of 24 months have been reported, and $8.6 \%$ of the patients in this study had received prior adjuvant chemotherapy [17]. A randomized phase III study has been performed looking at the use of GC and PCG for locally advanced or metastatic UC; the PCG response rate was $55.5 \%$, significantly higher than the $43.6 \%$ response rate with GC therapy [18]. In this study, the addition of paclitaxel to the GC combination provided a 3.1-month 


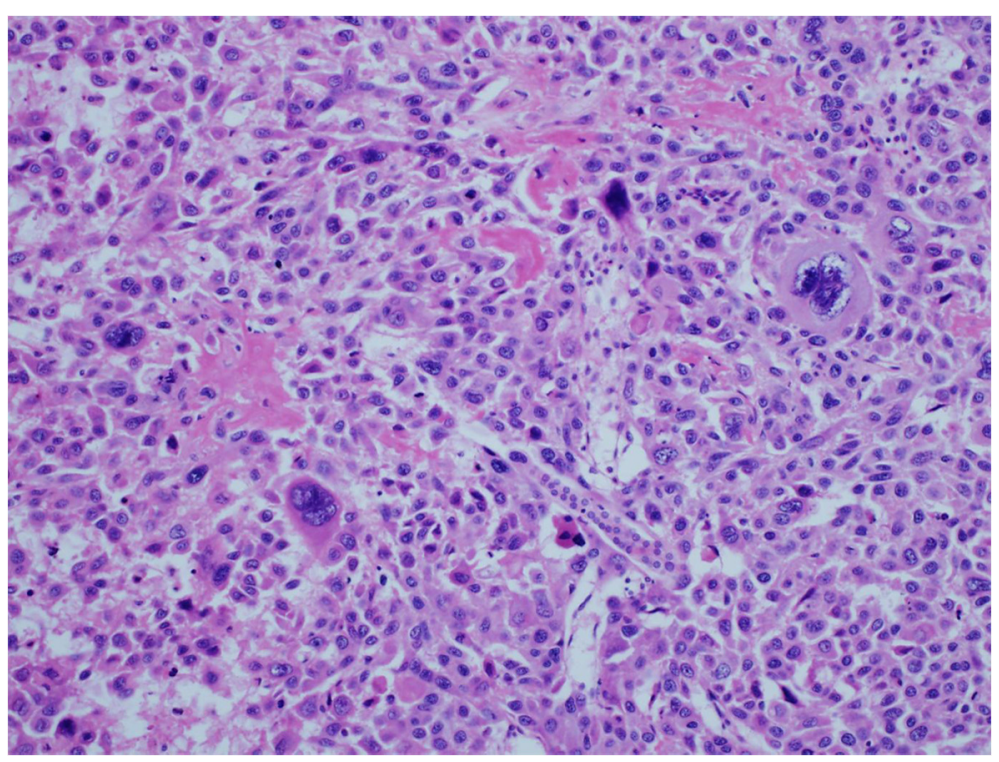

Figure 2 Hematoxylin \& eosin (HE) staining. Microscopic appearance showed urothelial carcinoma, grade3. Some of the nuclei were very large, irregular, and hyperchromatic.

survival benefit (15.8 months median overall survival for PCG versus 12.7 months for GC), but this did not reach significance. Our case report shows that patients can survive for a long-period of time after PCG treatment. Thus, PCG has potential as a novel first or second-line chemotherapy regimen for locally advanced or metastatic UC.
It is important to be able to predict which regimen is effective in patients with UC before the initiation of chemotherapy, and we should be able to avoid ineffective treatment. The survival rate of metastatic bladder cancer patients treated with either GC or PCG is significantly higher among patients with low ERCC1 levels
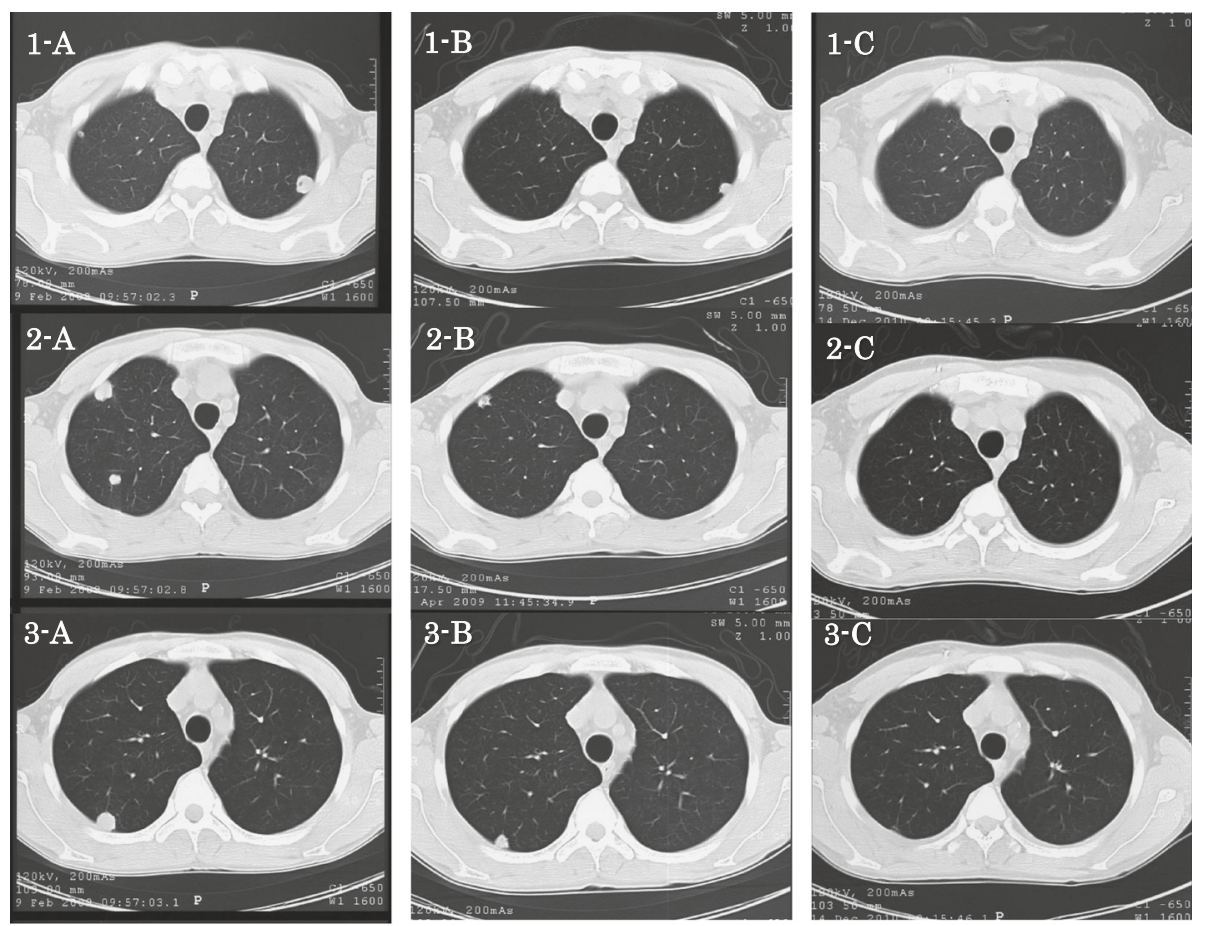

Figure 3 Three phases of chest CT scans showing the metastatic tumors. A, Before paclitaxel, cisplatin, and gemcitabine (PCG) chemotherapy. B, Reduction of the tumors after two courses of PCG. C, Disappearance of all metastatic tumors after seven courses of PCG. 
[19]. ERCC1 has been reported to be an independent prognosis prediction factor connected with platinumbased therapy for UC [19]. RRM1 has also been identified as a significant indicator of survival in patients receiving $\mathrm{GC}$, with low expression levels correlating with greater survival benefit [20]. However, biomarkers indicating which regimen, GC or PCG, was more effective were not expressed.

In this way, personalized medicine based on biomarkers has not been completely established yet, it would make a remarkable contribution to cancer therapy in the near future.

For dialysis patients, although it is possible to give GEM and PTX on non-hemodialysis days at the same dosage as in patients with normal renal function, it is necessary to reduce the dose of cisplatin by $50 \%$ to avoid nephrotoxicity; thus, hemodialysis initiation one hour after injection is recommended [21,22]. With these modifications, the plasma level of free cisplatin available for anti-tumor activity is almost the same as in patients with normal renal function [22]. There have been no reports of PCG treatment for hemodialysis patients; however, PCG was safely performed in our case.

\section{Conclusions}

The prognosis for metastatic UC is poor, with an average survival of less than six months for untreated patients [23]. Therefore, effective chemotherapy is needed for advanced or metastatic UC. This is the first report describing PCG combination therapy for metastatic UC arising in a transplanted renal allograft. PCG may have shown successful outcomes in our reported case, but further analyses on larger patient groups would be required to provide stronger evidence to support this therapeutic intervention.

\section{Consent}

Written informed consent was obtained from the patient for publication of this Case Report and any accompanying images. A copy of the written consent is available for review by the Editor-in-Chief of this journal.

\section{Abbreviations}

UC: Urothelial carcinoma; MVAC: Methotrexate/vinblastine/doxorubicin/ cisplatin; GC: Gemcitabine/cisplatin; PCG: Paclitaxel/cisplatin/gemcitabine; PTX: Paclitaxel; CDDP: Cisplatin; GEM: Gemcitabine.

\section{Competing interests}

The authors declare that they have no competing interests.

\section{Authors' contributions}

YK obtained consent from the patient, wrote the manuscript and made manuscript revisions. YT provided valuable help on the case report. $\mathrm{NI}$, and $\mathrm{MO}$ discussed the results and commented on the manuscript. ST and NN were responsible for critical revision of the manuscript. All authors read and approved the final manuscript.

\section{Author details}

'Department of Urology, Inoue Hospital, 16-17 Enoki-Cho, Suita 564-0053, Osaka, Japan. ${ }^{2}$ Department of Advanced Technology for Transplantation, Osaka University Graduate School of Medicine, Suita, Osaka, Japan.

${ }^{3}$ Department of Urology, Osaka University Graduate School of Medicine, Suita, Osaka, Japan.

Received: 20 October 2013 Accepted: 14 January 2015

Published online: 04 February 2015

\section{References}

1. Sternberg CN, Yagoda A, Scher HI, Watson RC, Herr HW, Morse MJ, et al. M-VAC (methotrexate, vinblastine, doxorubicin and cisplatin) for advanced transitional cell carcinoma of the urothelium. J Urol. 1988;139:461-9.

2. von der Maase H, Hansen SW, Roberts JT, Dogliotti L, Oliver T, Moore MJ, et al. Gemcitabine and cisplatin versus methotrexate, vinblastine, doxorubicine, and cisplatin in advanced or metastatic bladder cancer: results of a large, randomized, multinational, multicenter, phase III study. J Clin Oncol. 2000;17:3068-77.

3. Barrett WL, First MR, Aron BS, Penn I. Clinical course of malignancies in renal transplant recipients. Cancer. 1993;72:2186-9.

4. Navarro MD, López-Andréu M, Rodríguez-Benot A, Agüera ML, Del Castillo D, Aljama P. Cancer incidence and survival in kidney transplant patients. Transplant Proc. 2008:40:2936-40.

5. Miao Y, Everly JJ, Gross TG, Tevar AD, First MR, Alloway RR, et al. De novo cancers arising in organ transplant recipients are associated with adverse outcomes compared with the general population. Transplantation. 2009:87:1347-59.

6. Kasiske BL, Snyder JJ, Gilbertson DT, Wang C. Cancer after kidney transplantation in the United States. Am J Transplant. 2004;4:905-13.

7. Yuan M, Shi YB, Li ZH, Xia M, Ji GZ, Xu GX, et al. De novo urothelial carcinoma in kidney transplant patients with end-stage aristolochic acid nephropathy in China. Transplant Proc. 2009:41:1619-23.

8. Penn I. Primary kidney tumors before and after renal transplantation. Transplantation. 1995:59:480-5.

9. Melchior S, Franzaring L, Shardan A, Schwenke C, Plümpe A, Schnell R, et al. Urological de novo malignancy after kidney transplantation: a case for the urologist. J Urol. 2011;185:428-32.

10. Cox J, Colli JL. Urothelial cancers after renal transplantation. Int Urol Nephrol. 2011;43:681-6.

11. Jensen P, Ulhøi BP, Nielsen EH. Transitional cell carcinoma in a 25-year-old renal allograft. Nephrol Dial Transplant. 1998;13:3240-1.

12. Vervloessem I, Oyen R, Vanrenterghem $Y$, Van Poppel H, Van Hover P, Debakker $\mathrm{G}$, et al. Transitional cell carcinoma in a renal allograft. Eur Radiol. 1998:8:936-8.

13. Takaoka E, Miyazaki J, Kimura T, Kojima T, Kawai K, Murata Y, et al. Concurrent urothelial carcinoma in the renal pelvis of an allograft kidney and native recipient bladder: evidence of donor origin. Jpn J Clin Oncol. 2014:44:366-9

14. Tsaur I, Karalis A, Blaheta R, Juengel E, Vallo S, Scheuermann EH, et al. Transitional cell carcinoma of the native urinary tract after kidney transplantation: recommendations following a long-term retrospective analysis. Am J Med Sci. 2011;341:478-83.

15. Sternberg CN, Calabrò F, Pizzocaro G, Marini L, Schnetzer S, Sella A. Chemotherapy with an every-2-week regimen of gemcitabine and paclitaxel in patients with transitional cell carcinoma who have received prior cisplatin-based therapy. Cancer. 2001;92:2993-8.

16. Meluch AA, Greco FA, Burris 3rd HA, O'Rourke T, Ortega G, Steis RG, et al. Paclitaxel and gemcitabine chemotherapy for advanced transitional-cell carcinoma of the urothelial tract: a phase II trial of the Minnie pearl cancer research network. J Clin Oncol. 2001;19:3018-24.

17. Bellmunt J, Guillem V, Paz-Ares L, González-Larriba JL, Carles J, Batiste-Alentorn E, et al. Phase I-II study of paclitaxel, cisplatin, and gemcitabine in advanced transitional-cell carcinoma of the urothelium. Spanish Oncology Genitourinary Group. J Clin Oncol. 2000;18:3247-55.

18. Bellmunt J, von der Maase H, Mead GM, Skoneczna I, De Santis M, Daugaard G, et al. Randomized phase III study comparing paclitaxel/ cisplatin/gemcitabine and gemcitabine/cisplatin in patients with locally advanced or metastatic urothelial cancer without prior systemic therapy: EORTC Intergroup Study 30987. J Clin Oncol. 2012;30:1107-13. 
19. Bellmunt J, Paz-Ares L, Cuello M, Cecere FL, Albiol S, Guillem V, et al. Gene expression of ERCC1 as a novel prognostic marker in advanced bladder cancer patients receiving cisplatin-based chemotherapy. Ann Oncol. 2007;18:522-8.

20. Rosell R, Danenberg KD, Alberola V, Bepler G, Sanchez JJ, Camps C, et al. Ribonucleotide reductase messenger RNA expression and survival in gemcitabine/cisplatin-treated advanced non-small cell lung cancer patients. Clin Cancer Res. 2004;10:1318-25.

21. Ide H, Satou A, Hoshino K, Yasumizu Y, Uchida Y, Tasaka Y, et al. Successful management of metastatic urothelial carcinoma with gemcitabine and Paclitaxel chemotherapy in a hemodialysis patient. Urol Int. 2011;87:245-7.

22. Matsumoto K. Pharmacokinetics of cisplatin and methotrexate after M-VAC chemotherapy for advanced urothelial cancer in hemodialysis patients. Int J Urol. 2008;15:949-50

23. Ecke TH, Bartel P, Koch S, Ruttloff J, Theissig F. Chemotherapy with gemcitabine, paclitaxel, and cisplatin in the treatment of patients with advanced transitional cell carcinoma of the urothelium. Oncol Rep. 2006;16:1381-8

\section{Submit your next manuscript to BioMed Central and take full advantage of:}

- Convenient online submission

- Thorough peer review

- No space constraints or color figure charges

- Immediate publication on acceptance

- Inclusion in PubMed, CAS, Scopus and Google Scholar

- Research which is freely available for redistribution 